Review

\title{
Innate Immunity Derived Factors as External Modula- tors of the CXCLI2 - CXCR4 Axis and Their Role in Stem Cell Homing and Mobilization
}

\author{
Mariusz Z. Ratajczak ${ }^{1,2}$, Karol Serwin ${ }^{1}$, and Gabriela Schneider ${ }^{2}$ \\ 1. Stem Cell Biology Program at the James Graham Brown Cancer Center, University of Louisville, Louisville, Kentucky, USA. \\ 2. Department of Physiology Pomeranian Medical University, Szczecin, Poland.
}

\begin{abstract}
$\triangle$ Corresponding author: Prof. Mariusz Z. Ratajczak MD, PhD. Hoenig Endowed Chair, Professor and Director Stem Cell Institute at James Graham Brown Cancer Center, University of Louisville, 500 S. Floyd Street, Rm. 107, Louisville, KY 40202, USA. Tel: (502) 852-1788, Fax: (502) 852-3032 Email: mzrata01@louisville.edu.

(C) Ivyspring International Publisher. This is an open-access article distributed under the terms of the Creative Commons License (http://creativecommons.org/ licenses/by-nc-nd/3.0/). Reproduction is permitted for personal, noncommercial use, provided that the article is in whole, unmodified, and properly cited.
\end{abstract}

Received: 2012.05.20; Accepted: 2012.07.1 I; Published: 2013.01.12

\begin{abstract}
The $\alpha$-chemokine CXCLI2 (stromal derived factor-I; SDF-I) and its corresponding $\mathrm{G}_{\alpha \mid}$ protein-coupled CXCR4 receptor axis play an important role in retention of hematopoietic stem progenitor cells (HSPCs) in bone marrow (BM) stem cell niches. CXCLI2 has also been identified as a strong chemoattractant for HSPCs and implicated both in homing of HSPCs to BM after transplantation and in egress of these cells from BM into peripheral blood (PB). However, since CXCLI2, as a peptide, is highly susceptible to degradation by proteolytic enzymes, its real biological availability in biological fluids may be somewhat limited. In this review, we will present data demonstrating that the CXCLI2-CXCR4 axis is positively modulated by innate immunity-derived several external factors, ensuring that even low (near threshold) doses of CXCLI2 still exert a robust chemotactic influence on HSPCs.
\end{abstract}

Key words: CXCR4, CXCL12, SDF-1, C3a, LL-37, stem cell mobilization, stem cell homing.

\section{Introduction}

Several soluble factors secreted in the tissues that are endowed with chemotactic activity against a variety of hematopoietic cells, including neutrophils, monocytes, and lymphocytes, have been described [1-4]. By contrast, the number of factors that chemoattract hematopoietic stem progenitor cells (HSPCs) and thus regulate their trafficking in the living organism is quite limited [4-7]. The short list of HSPC chemoattractants that regulate two important aspects of HSPC trafficking, release from their niches in hematopoietic organs (a process known as mobilization) and homing back from circulation into these niches, includes i) $\alpha$-chemokine CXCL12 (stromal derived factor-1; SDF-1) [4, 8-10], ii) bioactive lipids, such as sphingosine-1-phosphate (S1P) and ceramide-1phosphate (C1P) [11-13], and iii) extracellular nucleotides, such as ATP and UTP $[14,15]$.

Chemokines are peptides that combine the properties of cytokines and chemotactic factors. While more than 50 different chemokines have been identified that bind to specific $G_{\alpha I}$ protein-coupled seven-transmembrane-spanning receptors present on the plasma membranes of target cells [16], CXCL12 is the only member of this family that chemoattracts HSPCs [17]. This may explain why the CXCL12-CXCR4 axis plays a crucial role in retention of HSPCs in bone marrow (BM). While CXCL12 has been reported to be highly expressed in the BM microenvironment [1, 2], 
the CXCR4 receptor is expressed both on short- and long-term repopulating HSPCs [4, 18]. Morphological studies have revealed that HSPCs are found in the BM microenvironment in so-called stem cell niches or in close contact with cells expressing high amounts of CXCL12, which are called CXCL12-abundant reticular (CAR) cells and which form a network of cells in the hematopoietic microenvironment [19]. Two types of stem cell niches have been identified: osteoblastic niches, lining trabecular bones, and endothelial niches, which are part of the hematopoietic endothelium in bone marrow sinusoids and are surrounded and connected by the abovementioned CAR cells [19]. CXCL12 is also secreted by BM stromal cells, including nestin-positive cells [20].

CXCL12 binds to the seven-transmembranespanning G protein-coupled CXCR4 receptor, and for many years this was considered to be the only receptor for this chemokine. Recent evidence, however, demonstrates that CXCL12 also binds to another $\mathrm{G}_{\alpha \mathrm{I}}$ protein-coupled seven-transmembrane-spanning receptor, CXCR7, and shares this receptor with another small chemokine, interferon-inducible T-cell chemoattractant (I-TAC) [21, 22]. However, since the I-TAC-CXCR7 axis does not play a significant or direct role in trafficking of normal HSPCs [21, 22], in this review we will focus on the CXCL12-CXCR4 axis. In particular, we will discuss the evidence that the biological activity of this axis is positively modulated by several external factors, including i) cationic anti-microbial peptides (CAMPs), ii) prostaglandin E2
(PGE2), iii) hyaluronic acid and fibrinogen fragments, iv) high-mobility group box 1 protein (HMGB1, also known as amphoterin), a non-histone chromosomal protein, as well as v) microvesicles released by activated cells. All of these positive regulators of the CXCL12-CXCR4 axis sensitize the responsiveness of HSPCs to an CXCL12 gradient by a variety of mechanisms (Figure 1).

\section{Hematopoietic stem progenitor cells (HSPCs) are nonstop travelers.}

Endowed with enhanced migratory properties, HSPCs migrate during organogenesis in the developing mammalian embryo, moving between major anatomical sites where hematopoiesis is initiated and/or is temporarily active (i.e., blood islands in the yolk sac, aortic endothelium, and fetal liver) before they reach their final destination in the developing hematopoietic microenvironment of BM in the third trimester of gestation [23]. Later in adult life, a small percentage of HSPCs is continuously released from $\mathrm{BM}$ niches into the $\mathrm{PB}$, which may be envisioned as a highway by which HSPCs relocate between hematopoietic stem cell niches in distant areas of BM in order to keep the total pool of stem cells in balance [23, 24]. The most important retention mechanism involves CXCL12, which is expressed in hematopoietic niches and interacts with its corresponding CXCR4 receptor expressed on HSPCs.

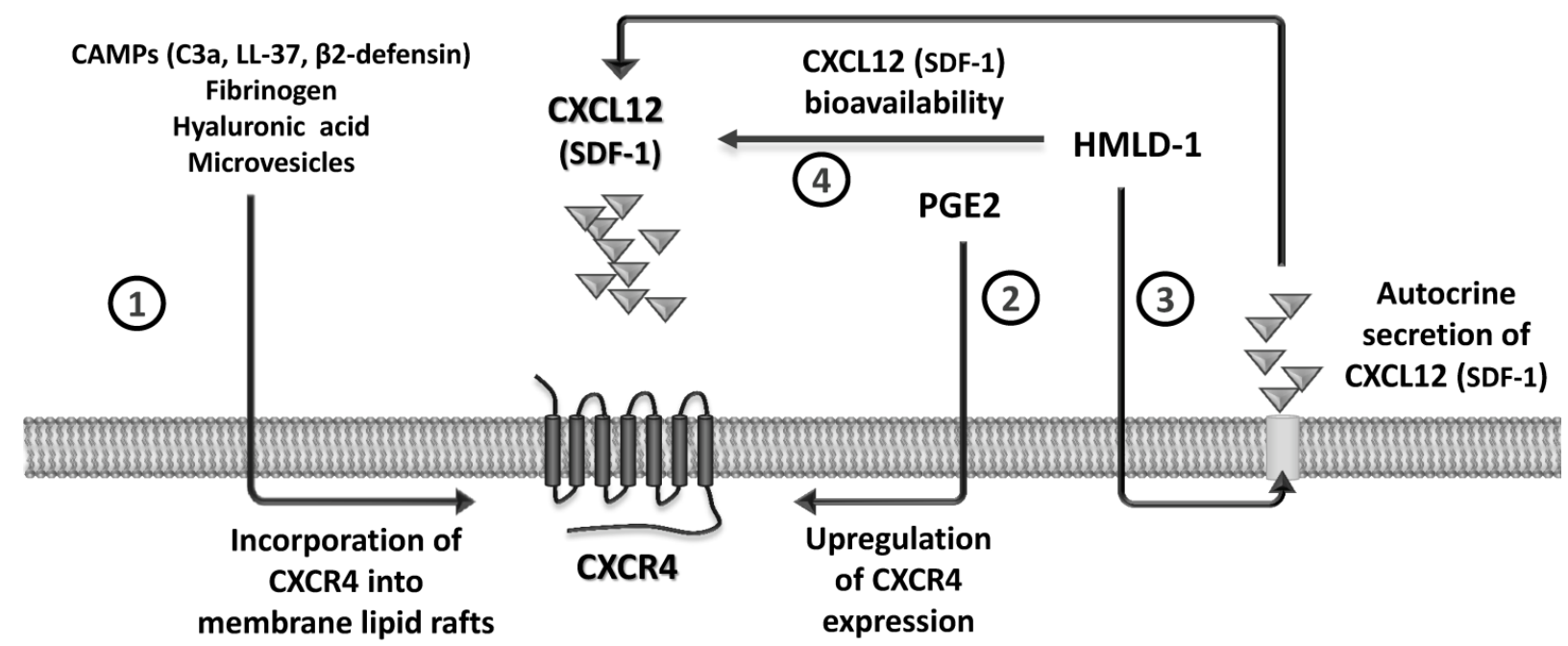

Figure I. Modulation of the CXCLI2-CXCR4 axis by external factors. The responsiveness of cells to a shallow CXCLI2 gradient is enhanced by several modulators. CC cleavage fragments C3a and des-Arg C3a, classical CAMPs (e.g., cathelicidin [LL-37] and $\beta 2$-defensin), fibrinogen, hyaluronic acid, and microvesicles promote incorporation of CXCR4 into membrane lipid rafts (I). PGE2 is a known upregulator of CXCR4 expression on the cell surface (2). The HMGBI effect is twofold, firstly by increasing autocrine secretion of CXCLI 2 by cells (3) and secondly by interacting with the CXCLI 2 molecule to form a heterocomplex that is a more efficient signaling ligand for CXCR4 (4). 
Pharmacologically enforced egress of HSPCs from $\mathrm{BM}$ into $\mathrm{PB}$, where the number of HSPCs circulating in PB increases up to 100 fold, is called "mobilization" [10, 25]. Several mobilizing drugs have been developed to interfere with mechanisms that promote retention of HSPCs in BM niches, including small-molecule antagonists of the CXCR4 chemokine receptor (e.g., AMD3100 or T140) $[12,26]$.

In a reverse mechanism, HSPCs home from PB to the BM. This is observed particularly after hematopoietic transplantation, where HSPCs infused intravenously migrate from $\mathrm{PB}$ into the $\mathrm{BM}$ microenvironment, settle into niches, and finally expand, leading to their engraftment. An important step that must precede hematopoietic transplantation is myeloablative conditioning by radio-chemotherapy of the transplant recipient. The main purpose of myeloblation before transplantation is destruction of the old hematopoiesis to make empty niches available for newly transplanted HSPCs $[4,8,9]$.

\section{The tissue level of CXCL12 and its sus- ceptibility to proteolytic enzymes.}

Expression of CXCL12 in the BM microenvironment is regulated at the transcriptional level by several factors, including hypoxia-inducible factor-1 alpha (HIF-1 $\alpha)$, which is upregulated, for example, after conditioning for transplantation by myeloablative treatment [27]. While it is relatively easy to measure the concentration of CXCL12 in biological fluids (e.g., PB plasma), measuring its level in the BM microenvironment is indirect, based on estimation of its concentration in BM aspirates. Similarly, it is currently impossible to measure CXCL12 concentration directly in BM sinusoids, and all measurements are performed on PB samples aspirated from large veins. These measurements, however, have revealed that CXCL12 is expressed in PB in the range $1-3 \mathrm{ng} / \mathrm{ml}$ [12], which is at least 100 times lower than in routine Transwell chemotaxis assays.

Despite upregulation of CXCL12 expression at the mRNA level in BM after conditioning for transplantation [28], the chemotactic homing gradient of CXCL12 protein originating in BM is attenuated by several proteolytic enzymes, whose expression is also induced in BM in parallel after myeloablative conditioning for transplantation $[29,30]$. It has been reported that metalloproteinase-2 (MMP-2), metalloproteinase-9 (MMP-9), cathepsin G, and elastase, which are secreted in the BM microenvironment from damaged/activated hematopoietic cells, remove a few $\mathrm{N}$-terminal amino acids from the CXCL12 peptide, resulting in a loss of chemotactic properties for this chemokine [29]. Interestingly, depending on the type of antibody employed for its detection, the CXCL12 peptide is still detectable by immunohistochemical staining or ELISA, but is not biologically active [29, 30].

Since myeloablative conditioning for hematopoietic transplantation by radio-chemotherapy induces a proteolytic microenvironment in $\mathrm{BM}$, the susceptibility of CXCL12 to proteolysis affects the CXCL12-mediated homing process of HSPCs after transplantation. To ameliorate this unwanted effect, several mechanisms have been identified that compensate for a decrease in the CXCL12 gradient, which are schematically depicted in Figure $\mathbf{1}$ and will be discussed below. These factors include elements of innate immunity, such as cleavage fragments of the third protein component of the CC ( $\mathrm{C} 3 \mathrm{a}$ and $\left.{ }_{\operatorname{desArg}} \mathrm{C} 3 \mathrm{a}\right)$ [31]; classical cationic antimicrobial peptides secreted by myeloid cells, such as cathelicidin (LL-37) and $\beta 2$-defensin [ 32, 33]; hyaluronic acid [34]; fibrinogen fragments [35]; high-mobility group box 1 protein (HMGB1, also known as amphoterin), a non-histone chromosomal protein [36, 37]; and microvesicles [38] released from cells damaged by myeloablative conditioning.

\section{Priming phenomena that sensitize the responsiveness of $\mathrm{CXCR4}{ }^{+} \mathrm{HSPC}$ to an CXCL12 gradient.}

As mentioned above, the biological activity of CXCL12 may decrease in BM due to the induction of a proteolytic microenvironment after conditioning for transplantation, as seen, for example, after lethal irradiation $[29,30]$. However, as shown in Figure 1, several factors are released from the damaged tissues that ameliorate this effect. These molecules are produced during the process of complement cascade (CC) activation, secreted from stromal and myeloid cells, or released from damaged cells, and cooperatively enhance or sensitize the responsiveness of HSPCs to a decreasing CXCL12 gradient. This process of sensitizing the responsiveness of cells to a shallow CXCL12 gradient in the presence of external modulatory molecules is known as the priming effect and can easily be evaluated in vitro in the Transwell migration assay, where two chambers (an upper chamber containing the tested cells and a lower chamber containing chemoattractant) are separated by a porous membrane that allows transmigration of cells that respond to the chemotactic gradient (Figure 2). Cells that respond to this gradient migrate and subsequently accumulate in the lower chamber. 

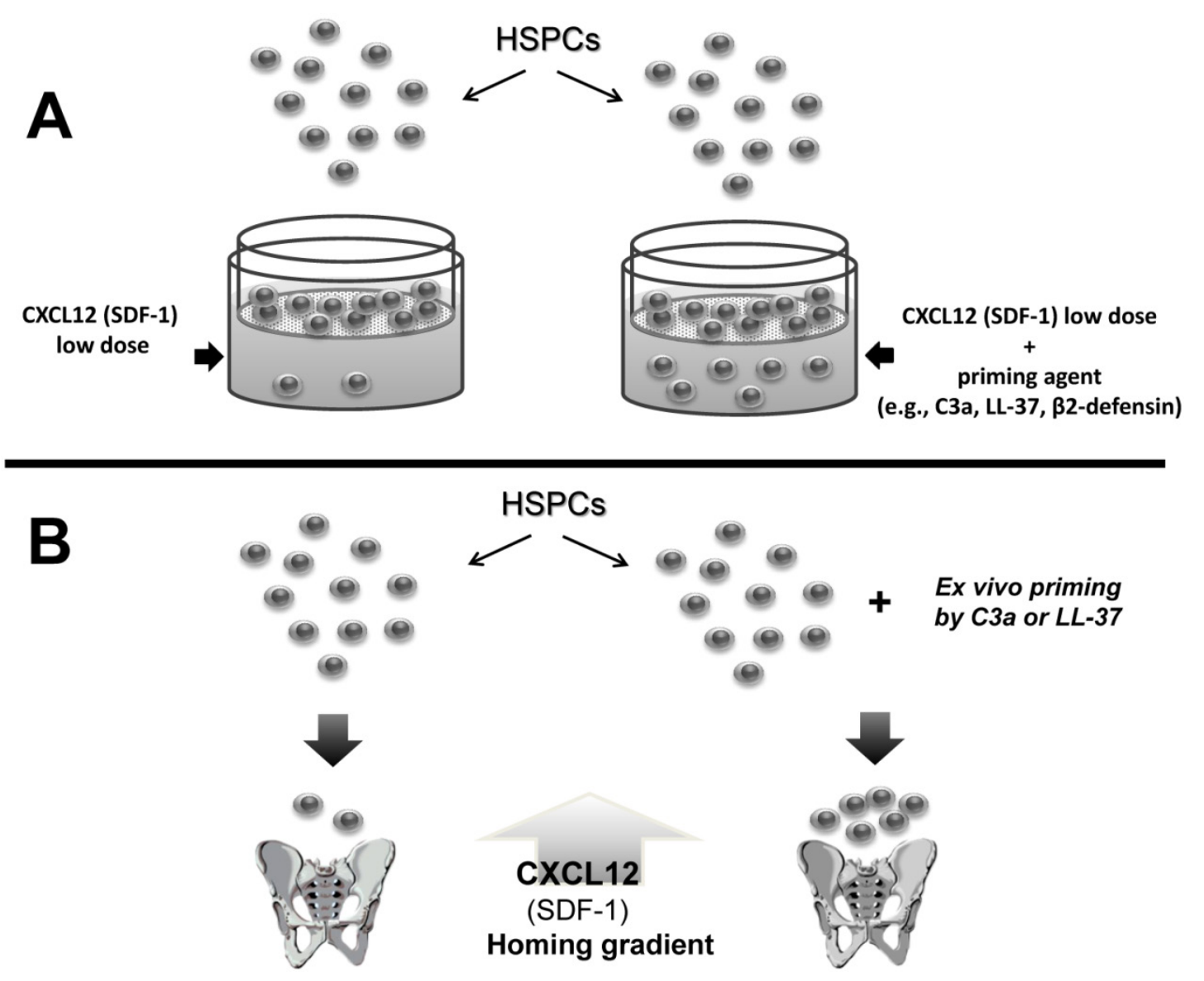

Figure 2. A priming effect increases the responsiveness of HSPCs to shallow CXCLI 2 gradients. The overall scheme of chemotactic assays performed in the Transwell system to evaluate the HSPC priming phenomenon (Panel A). In the presence of a priming agent (e.g., cationic antimicrobial peptides [CAMPs] such as C3a, cathelicidin [LL-37], or $\beta 2$-defensin), HSPCs respond more robustly to low doses of CXCLI2. This phenomenon is currently being tested in the clinic, where UCB is exposed ex vivo to a priming agent (e.g., C3a) before transplantation in order to respond more robust to CXCLI2 homing gradient (Panel B - thick arrow).

Figure 2 illustrates the findings that chemotaxis of HSPCs in response to a shallow CXCL12 gradient is significantly enhanced in the presence of several of these priming factors. This situation occurs also in vivo when the concentrations of these factors increases in a BM microenvironment damaged by myeloablative treatment and collectively enhance the responsiveness of transplanted HSPCs circulating in PB to an CXCL12 gradient [31-38].

- C3 cleavage fragments as priming factors. It has been demonstrated that the $\mathrm{CC}$, an evolutionarily ancient danger-sensing mechanism, becomes activated during conditioning for transplantation by radio- and chemotherapy $[29,31,32]$. The third component of the $\mathrm{CC}$ (C3) is an abundant protein in $\mathrm{PB}$ plasma $(1 \mathrm{mg} / \mathrm{ml})$ that becomes cleaved during CC activation by both classical and alternative pathways [39]. C3 cleavage leads to release of liquid-phase cleavage fragments, the $\mathrm{C} 3 \mathrm{a}$ and des-Arg $\mathrm{C} 3 \mathrm{a}$ anaphylatoxins [40]. C3a has a short half-life in plasma and is processed by serum carboxypeptidase $\mathrm{N}$ to des- $\mathrm{Arg} \mathrm{C} 3 \mathrm{a}$, which is a long-half-life cleavage product.

However, C3 cleavage fragments alone do not chemoattract HSPCs, our previous work on $\mathrm{C}^{-/-}$ mice revealed that animals lacking C3 display a significant delay in hematopoietic recovery from either sub-lethal irradiation or transplantation of wild type (WT) HSPCs [40]. Specifically, we observed that transplantation of histocompatible wild type (WT) Sca- $1^{+}$cells into $\mathrm{C}^{-/-}$mice resulted in a delay in hematological reconstitution in all hematopoietic lineages [40]. The fact that HSPCs from $\mathrm{C}^{-/-}$mice engrafted normally into irradiated WT mice suggests that there was a defect in the hematopoietic environment of $\mathrm{C}^{-/-}$mice and not an autonomous defect in the C3-/- mouse-derived HSPCs [40].

Since $\mathrm{C}^{-/-}$mice cannot activate or cleave $\mathrm{C} 3$, the $\mathrm{C} 3$ cleavage products $\mathrm{C} 3 \mathrm{a}$ and des-Arg $\mathrm{C} 3 \mathrm{a}$ were examined for a role in modulating the responsiveness of HSPCs to an CXCL12 homing gradient $[35,40]$. We 
observed that both short-half-life C3a and long-half-life des-ArgC $\mathrm{C}$ a significantly enhanced migration of HSPCs at a low or threshold level of CXCL12 in a Transwell migration assay [31]. The molecular explanation for this phenomenon has been identified as a C3a- and des-ArgC3a-mediated increase in CXCR4 incorporation into membrane lipid rafts $[35,40]$.

Lipid rafts are membrane domains rich in sphingolipids and cholesterol, which form a lateral assembly in a saturated glycerophospholipid environment of cell surface membranes. The raft domains are known to serve as moving platforms on the cell and are also good sites for crosstalk between various cell surface molecules (e.g., CXCR4) and proteins that form intra-cellular signaling pathways. For example, it has recently been reported that small guanine nucleotide triphosphatases (GTPases), such as Rac-1 and Rac-2, which are crucial for engraftment of hematopoietic cells after transplantation, are associated with lipid rafts on migrating HSPCs [41-45]. Therefore, since the CXCR4 receptor is a lipid raft-associated protein, its signaling ability is enhanced if it is incorporated into membrane lipid rafts, where it can better interact with several signaling molecules, including the small GTPase Rac-1. This co-localization of CXCR4 and Rac-1 in lipid rafts facilitates GTP binding and activation of Rac-1 [35]. Thus, the generation of C3 cleavage fragments in the BM microenvironment may somehow act as a protective mechanism that increases the responsiveness of HSPCs to an CXCL12 gradient when it is degraded by a proteolytic microenvironment after myeloablative conditioning for transplantation [29, 30]. In C3-deficient mice this phenomenon is attenuated, explaining why these animals show delayed engraftment after hematopoietic transplantation.

In this context, activation of the $\mathrm{CC}$, which leads to increases in $\mathrm{C} 3 \mathrm{a}$ or des- $\mathrm{Arg} \mathrm{C} 3 \mathrm{a}$ levels in $\mathrm{BM}$ after myeloablative conditioning [29], can be envisioned as one of the mechanisms that promotes homing of HSPCs (Figures 1-2). In fact, this relatively simple strategy for enhancing responsiveness of the HSPCs to be transplanted, which is based on short ex vivo exposure of HSPCs before transplantation to C3a, has been proposed for use in the clinic to enhance engraftment of HSPCs, and an appropriate FDA-approved clinical trial has been initiated for patients undergoing umbilical cord blood (UCB) transplantations.

- Cationic antimicrobial peptides (CAMPs) as priming factors. CAMPs are host-defense peptides and are an evolutionarily conserved component of the innate immune response [46-48]. Interestingly, the C3a and des-Arg $\mathrm{C} 3 \mathrm{a}$ anaphylatoxins mentioned above share several properties with classical peptides from the CAMP family [47]. CAMPs have been demonstrated to perforate prokaryotic cell membranes and thus kill bacteria, enveloped viruses, and fungi, but affect only the organization and not the viability of eukaryotic cell membranes. The selective effects of these natural antibiotics (i.e., prokaryotic killing and eukaryotic membrane perturbation) are known to be dependent on the characteristics of cell membranes [46-48]. While prokaryotic cell membranes are susceptible to strong electrostatic and hydrophobic interactions with these "natural antibiotics", which may lead to membrane perforation, the cell membranes of eukaryotic cells are more resistant to the toxic effects of these peptides, because of their high cholesterol content and weak hydrophobic interactions with these peptides [46-48].

One of the interesting properties of CAMPs that we identified is their ability to enhance or prime the responsiveness of cells to an CXCL12 gradient (Figure 1). Therefore, cathelicidin (LL-37) and $\beta 2$-defensin, which, like C3a, belong to the CAMP family, positively prime the responsiveness of HSPCs to an CXCL12 homing gradient (Figures 1-2) [31-33].

Like C3 cleavage fragments, CAMPs also enhance incorporation of CXCR4 into lipid rafts so that CXCR4 is activated more efficiently in the presence of low doses of CXCL12, which facilitates its signaling. Interestingly, LL-37 and $\beta-2$ defensin, first described as being secreted by myeloid cells, are also secreted by BM stromal cells and, like CXCL12, their expression is regulated by HIF-1 $\alpha$ [48]. However, while LL-37 and $\beta-2$ defensin are also peptides, they seem to be more resistant to proteolytic enzymes and are thus more stable in a proteolytic microenvironment than CXCL12.

- Other priming molecules. In addition to the CAMPs, hylauronic acid [34], fibrinogen [35], and cell membrane-derived microvesicles [38] have also been reported to increase the responsiveness of HSPCs to an CXCL12 gradient. The exact mechanism of this priming effect, however, has still not been elucidated, but is most likely mediated by the interaction of these factors with integrin receptors on HSPCs. Activation of these receptors may promote lipid raft formation, again leading to incorporation of CXCR4 into lipid rafts [35]. This putative phenomenon has been confirmed for fibrinogen $[35,40]$ and the small spherical membrane fragments shed and secreted from the activated cells called microvesicles $[35,38]$.

Thus, incorporation of CXCR4 into membrane lipids rafts, which ensures its most optimal signaling, is a mechanism shared by several factors that positively modulate the CXCL12-CXCR4 axis, including 
CC cleavage fragments, CAMPs, and molecules related to coagulation/inflammation (e.g., fibrinogen and hyaluronic acid).

That priming molecules (e.g., C3 cleavage fragment, CAMPs, and microvesicles) are generated during harvest of mobilized HSPCs from PB during the process of leucophoresis, may explain why, in certain situations, mobilized PB HSPCs engraft faster than HSPCs harvested by aspiration under steady-state conditions BM [49]. This mechanism, which is involved in trafficking of CXCR4 ${ }^{+}$HSPCs, may also play an important role in migration of other types of $\mathrm{CXCR}^{+}$cells, such as lymphocytes or macrophages in tissues affected by inflammation.

\section{Prostaglandin E (PGE2) increases the homing activity of the CXCL12-CXCR4 axis}

In addition to the abovementioned CC-released and CAMP-related priming factors, PGE2 has also been purported to increase the responsiveness of HSPCs to an CXCL12 gradient [50]. This important effect of PGE2 is particularly observable in the most primitive HSPCs $[6,51]$. However, the mechanism of the PGE2 effect on this process is not lipid raft-mediated (Figure 1). Instead, PGE2 plays an important role in homing of HSPCs by upregulating the expression of CXCR4 on the surface of HSPCs, and this seems to be the most likely mechanism responsible for increasing chemotaxis in response to an CXCL12 gradient after pretreatment of HSPCs with PGE2 [6, 51, 52].

In further support of this notion, the positive effect of PGE2 on migration of HSPCs in response to an CXCL12 gradient was blocked by a selective CXCR4 antagonist (AMD3100), indicating a primary role for CXCR4 in this process. Furthermore, it has been shown that, while PGE2 upregulates CXCR4 in microvascular endothelial cells by involving the Sp1 transcription factor [52], in hematopoietic cells this PGE2 effect is mediated by the PKC-zeta signaling pathway [50].

It has been demonstrated in a murine model that HSPCs cultured with PGE2 before transplantation engraft better in lethally irradiated recipients [51]. Thus, as with C3a, PGE2 is currently employed in clinical trials as an ex vivo priming agent to enhance the responsiveness of HSPCs to be transplanted to an CXCL12 gradient.

In further support of a role for PGE2 in homing of transplanted HSPCs, it has recently been reported that the level of this eicosanoid is significantly upregulated in BM conditioned for hematopoietic trans- plantation by lethal irradiation [29]. Thus, PGE2, together with $\mathrm{C} 3 \mathrm{a}$ and CAMPs, is released in the BM microenviroment, and all these factors cooperatively enhance the homing responsiveness of transplanted HSPCs.

In addition to PGE2, other factors have also been described that may modulate expression of CXCR4 on cells, such as glucocorticoids [53], lysophosphatidylcholine [54], TGF- $\beta 1$ [55], VEGF [56], IFN-a [57], and several interleukins (IL-2, IL-4, and IL-7) [58]. These factors however, were not tested for whether they modulate the responsiveness of HSPCs to an CXCL12 gradient.

\section{The positive effect of HMGB1 on the chemotactic responsiveness of cells to CXCL12.}

The high-mobility group box 1 protein (HMGB1, also known as amphoterin) is a non-histone chromosomal protein that modulates CXCL12-mediated migration of hematopoietic cells, as recently demonstrated [36, 37]. This chromatin architectural protein belongs to broad family of so-called "alarmins" and is released from damaged cells, for example, in the BM microenvironment after myeloablative conditioning for transplantation.

As depicted in Figure 1, the twofold HMGB1 effect is by i) forming a heterocomplex with the CXCL12 molecule to present it to the CXCR4 ${ }^{+}$cells as a more efficient signaling ligand [36] and ii) sustaining autocrine secretion of CXCL12 [37]. So far, these mechanisms have been demonstrated for migrating monocytes [36] and murine BM-derived macrophages [37], respectively. In the first case, fluorescence resonance energy transfer revealed that the HMGB1-CXCL12 complex promotes different conformational rearrangements after binding to CXCR4 than when CXCL12 alone interacts with CXCR4 [36].

In the second case, it was found that the HMGB1-induced NF- $\kappa B$ noncanonical pathway is solely required to maintain the autocrine CXCL12-CXCR4 loop involved in migration of murine macrophages. However, since HSPCs also secrete autocrine CXCL12 [59], it would be interesting to test the relevance of HMGB1 to the migration of HSPCs as well.

\section{Future directions}

As demonstrated in this review, evidence has accumulated that the chemotactic responsiveness of HSPCs to several different types of homing gradients can be modulated by ex vivo manipulations. One potential strategy is to take advantage of the 
HSPC-priming approach. For instance, the possibility of accelerating or enhancing the homing of HSPCs by ex vivo exposure of cells to C3a before infusion into the patient is currently being evaluated in an ongoing clinical trial (Masonic Cancer Center, University of Minnesota). Another interesting molecule that should be tested in the clinical setting as a potential priming factor is the cathelicidin LL-37. The advantage of LL-37 is that it is a physiological factor secreted by BM stromal cells and is a more potent priming factor than C3a [33].

Another possible ex vivo manipulation of HSPCs in grafting is exposure to PGE2 in order to upregulate expression of CXCR4 to enhance the homing of transplanted cells [50-52]. This strategy is also currently being evaluated in a clinical trial. Overall, these strategies are particularly valuable in clinical situations where the number of HSPCs available to be transplanted is limited, as seen, for example, in UCB transplants.

In addition to the abovementioned strategies, there are other possibilities for manipulating HSPCs to enhance their homing that are beyond the scope of this review. For example, based on observations that the CXCL12 interaction with CXCR4 ${ }^{+}$HSPCs is attenuated by the dipeptidyl-peptidase CD26, inhibition of CD26 on HSPCs could enhance their chemotactic responsiveness to an CXCL12 gradient $[60,61]$. Another interesting strategy is modification of adhesion molecules on HSPCs by ex vivo treatment with fucosyltransferase, which increases the level of fucosylation of these receptors [62]. As has demonstrated, after blockage of CD26 or after fucosylation of adhesion molecules, human HSPCs home and subsequently engraft better in immunodeficient mice [62].

Moreover, evidence accumulates that different subsets of stem cells in BM may be preferentially mobilized by different factors. To support this vascular endothelial growth factor (VEGF) mobilizes preferentially endothelial progenitor cells [63].

These developments suggest that hematological transplantology can take advantage of basic research to translate these observations into more efficient clinical protocols.

\section{Acknowledgments}

This work was supported by NIH grant 2R01 DK074720, Stella and Henry Endowment, the European Union structural funds (Innovative Economy Operational Program POIG.01.01.02-00-109/09-00) and grant Maestro 2011/02/A/NZ4/00035.

\section{Competing Interests}

The authors have declared that no competing interest exists.

\section{References}

1. Nagasawa T, Hirota S, Tachibana K, Takakura N, Nishikawa S, Kitamura $\mathrm{Y}$, et al. Defects of B-cell lymphopoiesis and bone-marrow myelopoiesis in mice lacking the CXC chemokine PBSF/SDF-1. Nature. 1996; 382: 635-8.

2. Tachibana K, Hirota S, Iizasa H, Yoshida H, Kawabata K, Kataoka Y, et al. The chemokine receptor CXCR4 is essential for vascularization of the gastrointestinal tract. Nature. 1998; 393: 591-4

3. Pelus LM, Fukuda S. Chemokine-mobilized adult stem cells; defining a better hematopoietic graft. Leukemia. 2008; 22: 466-73.

4. Lapidot T, Dar A, Kollet O. How do stem cells find their way home? Blood. 2005; 106: 1901-10.

5. Massberg S, Schaerli P, Knezevic-Maramica I, Kollnberger M, Tubo N, Moseman EA, et al. Immunosurveillance by hematopoietic progenitor cells trafficking through blood, lymph, and peripheral tissues. Cell. 2007; 131: 994-1008.

6. Hoggatt J, Pelus LM. Eicosanoid regulation of hematopoiesis and hematopoietic stem and progenitor trafficking. Leukemia. 2010; 24: 1993-2002.

7. Ratajczak MZ, Kim CH, Abdel-Latif A, Schneider G, Kucia M, Morris AJ, et al. A novel perspective on stem cell homing and mobilization: review on bioactive lipids as potent chemoattractants and cationic peptides as underappreciated modulators of responsiveness to SDF-1 gradients. Leukemia. 2012; 26: 63-72.

8. Levesque JP, Helwani FM, Winkler IG. The endosteal 'osteoblastic' niche and its role in hematopoietic stem cell homing and mobilization. Leukemia. 2010; 24: 1979-92.

9. Doan PL, Chute JP. The vascular niche: home for normal and malignant hematopoietic stem cells. Leukemia. 2012; 26: 54-62.

10. Greenbaum AM, Link DC. Mechanisms of G-CSF-mediated hematopoietic stem and progenitor mobilization. Leukemia. 2011; 25: 211-7.

11. Kimura T, Boehmler AM, Seitz G, Kuci S, Wiesner T, Brinkmann V, et al. The sphingosine 1-phosphate receptor agonist FTY720 supports CXCR4-dependent migration and bone marrow homing of human CD34+ progenitor cells. Blood. 2004; 103: 4478-86.

12. Ratajczak MZ, Lee H, Wysoczynski M, Wan W, Marlicz W, Laughlin MI, et al. Novel insight into stem cell mobilization-plasma sphingosine-1-phosphate is a major chemoattractant that directs the egress of hematopoietic stem progenitor cells from the bone marrow and its level in peripheral blood increases during mobilization due to activation of complement cascade/membrane attack complex. Leukemia. 2010; 24: 976-85.

13. Golan K, Vagima Y, Ludin A, Itkin T, Cohen-Gur S, Kalinkovich A, et al. S1P promotes murine progenitor cell egress and mobilization via S1P1-mediated ROS signaling and SDF-1 release. Blood. 2012; 119: 2478-88.

14. Lemoli RM, Ferrari D, Fogli M, Rossi L, Pizzirani C, Forchap S, et al. Extracellular nucleotides are potent stimulators of human hematopoietic stem cells in vitro and in vivo. Blood. 2004; 104: 1662-70.

15. Rossi L, Manfredini R, Bertolini F, Ferrari D, Fogli M, Zini R, et al. The extracellular nucleotide UTP is a potent inducer of hematopoietic stem cell migration. Blood. 2007; 109: 533-42.

16. Zlotnik A, Yoshie O, Nomiyama H. The chemokine and chemokine receptor superfamilies and their molecular evolution. Genome Biology. 2006; 7: 243-51.

17. Wright DE, Bowman EP, Wagers AJ, Butcher EC, Weissman IL. Hematopoietic stem cells are uniquely selective in their migratory response to chemokines. J Exp Med. 2002; 195: 1145-54.

18. Vagima Y, Lapid K, Kollet O, Goichberg P, Alon R, Lapidot T. Pathways implicated in stem cell migration: the SDF-1/CXCR4 axis. Methods Mol Biol. 2011; 750: 277-89

19. Sugiyama T, Kohara H, Noda M, Nagasawa T. Maintenance of the hematopoietic stem cell pool by CXCL12-CXCR4 chemokine signaling in bone marrow stromal cell niches. Immunity. 2006; 25: 977-88.

20. Mendez-Ferrer S, Michurina TV, Ferraro F, Mazloom AR, Macarthur BD, Lira SA, et al. Mesenchymal and haematopoietic stem cells form a unique bone marrow niche. Nature. 2010, 466: 829-34.

21. Hartmann TN, Grabovsky V, Pasvolsky R, Shulman Z, Buss EC, Spiegel A, et al. A crosstalk between intracellular CXCR7 and CXCR4 involved in rapid CXCL12-triggered integrin activation but not in chemokine-triggered motility of human T lymphocytes and CD34+ cells. J Leuk Biol. 2008; 84: 1130-40.

22. Tarnowski M, Liu R, Wysoczynski M, Ratajczak J, Kucia M, Ratajczak MZ. CXCR7: a new SDF-1-binding receptor in contrast to normal 
CD34(+) progenitors is functional and is expressed at higher level in human malignant hematopoietic cells. Eur J Haeamtol. 2010, 85: 472-83.

23. Mikkola HK, Orkin SH. The journey of developing hematopoietic stem cells. Development 2006; 133: 3733-44.

24. Ratajczak MZ, Kim CH, Wojakowski W, Janowska-Wieczorek A, Kucia M, Ratajczak J. Innate immunity as orchestrator of stem cell mobilization. Leukemia. 2010; 24: 1667-75.

25. Ratajczak MZ. Spotlight series on stem cell mobilization: many hands on the ball, but who is the quarterback? Leukemia. 2010; 24: 1665-6.

26. Rettig MP, Ansstas G, DiPersio JF. Mobilization of hematopoietic stem and progenitor cells using inhibitors of CXCR4 and VLA-4. Leukemia 2012; 26: 34-53.

27. Ceradini DJ, Kulkarni AR, Callaghan MJ, Tepper OM, Bastidas N, Kleinman ME et al.. Progenitor cell trafficking is regulated by hypoxic gradients through HIF-1 induction of SDF-1. Nat Med. 2004; 10: 858-864

28. Peled A, Grabovsky V, Habler L, Sandbank J, Arenzana-Seisdedos F, Petit I, et al. The chemokine SDF-1 stimulates integrin-mediated arrest of CD34(+) cells on vascular endothelium under shear flow. J Clin Invest. 1999; 104: 1199-211.

29. Kim CH, Wu W, Wysoczynski M, Abdel-Latif A, Sunkara M, Morris A, et al. Conditioning for hematopoietic transplantation activates the complement cascade and induces a proteolytic environment in bone marrow: a novel role for bioactive lipids and soluble $\mathrm{C} 5 \mathrm{~b}-\mathrm{C} 9$ as homing factors. Leukemia 2012; 26: 106-16.

30. Cho SY, Xu M, Roboz J, Lu M, Mascarenhas J, Hoffman R. The effect of CXCL12 processing on CD34+ cell migration in myeloproliferative neoplasms. Cancer Res. 2010; 70: 3402-10.

31. Reca R, Mastellos D, Majka M, Marquez L, Ratajczak J, Franchini S, et al. Functional receptor for $\mathrm{C} 3 \mathrm{a}$ anaphylatoxin is expressed by normal hematopoietic stem/progenitor cells, and C3a enhances their homing-related responses to SDF-1. Blood 2003; 101: 3784-93.

32. Lee HM, Wu W, Wysoczynski M, Liu R, Zuba-Surma EK, Kucia M, et al. Impaired mobilization of hematopoietic stem/progenitor cells in C5-deficient mice supports the pivotal involvement of innate immunity in this process and reveals novel promobilization effects of granulocytes. Leukemia 2009; 23: 2052-62.

33. Wu W, Kim CH, Liu R, Kucia M, Marlicz W, Greco N, et al. The bone marrow-expressed antimicrobial cationic peptide LL-37 enhances the responsiveness of hematopoietic stem progenitor cells to an SDF-1 gradient and accelerates their engraftment after transplantation. Leukemia 2012; 26: 736-45.

34. Avigdor A, Goichberg P, Shivtiel S, Dar A, Peled A, Samira S, et al. CD44 and hyaluronic acid cooperate with SDF-1 in the trafficking of human CD34+ stem/progenitor cells to bone marrow. Blood 2004; 103: 2981-9.

35. Wysoczynski M, Reca R, Ratajczak J, Kucia M, Shirvaikar N, Honczarenko M, et al. Incorporation of CXCR4 into membrane lipid rafts primes homing-related responses of hematopoietic stem/progenitor cells to an SDF-1 gradient. Blood 2005; 105: 40-8.

36. Kew RR, Penzo M, Habiel DM, Marcu KB. The IKKalpha-dependent NF-kappaB p52/RelB noncanonical pathway is essential to sustain a CXCL12 autocrine loop in cells migrating in response to HMGB1. J Immunol. 2012; 188: 2380-6.

37. Schiraldi M, Raucci A, Munoz LM, Livoti E, Celona B, Venereau E, et al. HMGB1 promotes recruitment of inflammatory cells to damaged tissues by forming a complex with CXCL12 and signaling via CXCR4. J Exp Med. 2012; 209: 551-63.

38. Janowska-Wieczorek A, Majka M, Kijowski J, Baj-Krzyworzeka M, Reca $\mathrm{R}$, Turner AR, et al. Platelet-derived microparticles bind to hematopoietic stem/progenitor cells and enhance their engraftment. Blood 2001; 98: 3143-9.

39. Ricklin D, Hajishengallis G, Yang K, Lambris JD. Complement: a key system for immune surveillance and homeostasis. Nat Immunol. 2010; 11: 785-797.

40. Ratajczak MZ, Reca R, Wysoczynski M, Kucia M, Baran JT, Allendorf DJ, et al. Transplantation studies in C3-deficient animals reveal a novel role of the third complement component (C3) in engraftment of bone marrow cells. Leukemia 2004; 18: 1482-90.

41. Gomez-Mouton C, Lacalle RA, Mira E, Jimenez-Baranda S, Barber DF, Carrera AC, et al. Dynamic redistribution of raft domains as an organizing platform for signaling during cell chemotaxis. J Cell Biol. 2004; 164: 759-68.

42. Guan JL. Cell biology. Integrins, rafts, Rac, and Rho. Science 2004; 303: 773-4.

43. Cancelas JA, Lee AW, Prabhakar R, Stringer KF, Zheng Y, Williams DA. Rac GTPases differentially integrate signals regulating hematopoietic stem cell localization. Nature Med. 2005; 11: 886-91.
44. Gu Y, Filippi MD, Cancelas JA, Siefring JE, Williams EP, Jasti AC, et al. Hematopoietic cell regulation by Rac1 and Rac2 guanosine triphosphatases. Science 2003; 302: 445-9.

45. Wysoczynski M, Reca R, Lee H, Wu W, Ratajczak J, Ratajczak MZ. Defective engraftment of C3aR-/- hematopoietic stem progenitor cells shows a novel role of the C3a-C3aR axis in bone marrow homing. Leukemia 2009; 23: 1455-61.

46. Ganz T. Defensins: antimicrobial peptides of innate immunity. Nature Rev. 2003; 3: 710-20.

47. Malmsten M, Schmidtchen A. Antimicrobial C3a--biology, biophysics, and evolution. Adv Exp Med \& Biol. 2007; 598: 141-58.

48. Bucki R, Leszczynska K, Namiot A, Sokolowski W. Cathelicidin LL-37: a multitask antimicrobial peptide. Arch Immunol Ther Exp. 2010; 58: $15-25$.

49. Anderson D, DeFor T, Burns L, McGlave P, Miller J, Wagner J, et al. A comparison of related donor peripheral blood and bone marrow transplants: importance of late-onset chronic graft-versus-host disease and infections. Biol Blood Marrow Transplant. 2003; 9: 52-9.

50. Goichberg P, Kalinkovich A, Borodovsky N, Tesio M, Petit I, Nagler A, et al. cAMP-induced PKCzeta activation increases functional CXCR4 expression on human CD34+ hematopoietic progenitors. Blood. 2006; 107: 870-9.

51. Hoggatt J, Singh P, Sampath J, Pelus LM. Prostaglandin E2 enhances hematopoietic stem cell homing, survival, and proliferation. Blood. 2009; 113: 5444-55.

52. Pelus LM, Hoggatt J. Pleiotropic effects of prostaglandin E2 in hematopoiesis; prostaglandin E2 and other eicosanoids regulate hematopoietic stem and progenitor cell function. Prostaglandins \& Other Lipid Mediators. 2011; 96: 3-9.

53. Curnow SJ, Wloka K, Faint JM, Amft N, Cheung CM, Savant V, et al. Topical glucocorticoid therapy directly induces up-regulation of functional CXCR4 on primed T lymphocytes in the aqueous humor of patients with uveitis. J Immunol. 2004; 172: 7154-61.

54. Han KH, Hong KH, Ko J, Rhee KS, Hong MK, Kim JJ, et al. Lysophosphatidylcholine up-regulates CXCR4 chemokine receptor expression in human CD4 T cells. J Leuk Biol. 2004; 76: 195-202.

55. Franitza S, Kollet O, Brill A, Vaday GG, Petit I, Lapidot T, et al. TGF-beta1 enhances SDF-1alpha-induced chemotaxis and homing of naive $\mathrm{T}$ cells by up-regulating CXCR4 expression and downstream cytoskeletal effector molecules. Eur J Immunol. 2002; 32: 193-202.

56. Bachelder RE, Wendt MA, Mercurio AM. Vascular endothelial growth factor promotes breast carcinoma invasion in an autocrine manner by regulating the chemokine receptor CXCR4. Cancer Res. 2002; 62: 7203-6.

57. Yonezawa A, Morita R, Takaori-Kondo A, Kadowaki N, Kitawaki T, Hori T, et al. Natural alpha interferon-producing cells respond to human immunodeficiency virus type 1 with alpha interferon production and maturation into dendritic cells. J Virol. 2003; 77: 3777-84.

58. Iikura M, Miyamasu M, Yamaguchi M, Kawasaki H, Matsushima K, Kitaura M, et al. Chemokine receptors in human basophils: inducible expression of functional CXCR4. J Leuk Biol. 2001; 70: 113-20.

59. Lataillade JJ, Clay D, Bourin P, Herodin F, Dupuy C, Jasmin C, et al. Stromal cell-derived factor 1 regulates primitive hematopoiesis by suppressing apoptosis and by promoting $\mathrm{G}(0) / \mathrm{G}(1)$ transition in CD34(+) cells: evidence for an autocrine/paracrine mechanism. Blood. 2002; 99: 1117-29.

60. Christopherson KW, 2nd, Hangoc G, Mantel CR, Broxmeyer HE. Modulation of hematopoietic stem cell homing and engraftment by CD26. Science 2004; 305: 1000-3.

61. Peranteau WH, Endo M, Adibe OO, Merchant A, Zoltick PW, Flake AW. CD26 inhibition enhances allogeneic donor-cell homing and engraftment after in utero hematopoietic-cell transplantation. Blood. 2006; 108: 4268-74.

62. Xia L, McDaniel JM, Yago T, Doeden A, McEver RP. Surface fucosylation of human cord blood cells augments binding to P-selectin and E-selectin and enhances engraftment in bone marrow. Blood. 2004; 104: 3091-6.

63. Pitchford SC, Furze RC, Jones CP, Wengner AM, Rankin SM. Differential mobilization of subsets of progenitor cells from the bone marrow. Cell Stem Cell. 2009; 4:62-72. 